\title{
The effect of green tea on the shear strength of brackets after home whitening treatment
}

\author{
Renata C. A. Schwertner ${ }^{1}$, Joyce S. Y. Leoncio' ${ }^{\text {, Alessandro Schwertner }}{ }^{2}$, Ricardo D. Guiraldo ${ }^{1}$, Murilo B. Lopes ${ }^{\text {, }}$ \\ Hellen C. De Carvalho ${ }^{1}$, Alcides Gonini-Júnior ${ }^{1}$ and Sandrine B. Berger ${ }^{1 *}$
}

*Correspondence:

berger.sandrine@gmail.com

${ }^{1}$ Department of Restorative Dentistry, University of North

Parana, Rua Marselha,

183 Jardim Piza, Londrina,

PR 86041-120, Brazil

Full list of author information

is available at the end of the

article

\begin{abstract}
This study, we evaluated the effect of green tea on the shear bond strength of metal orthodontic brackets to human enamel after home whitening treatment. Ninety premolars were selected and divided into six experimental groups: G1, no treatment (positive control); G2, whitening (negative control); G3, no whitening $+10 \%$ sodium ascorbate (SA) gel; G4, no whitening + $10 \%$ green tea (GT) gel; G5, whitening + SA; and $\mathrm{G} 6$, whitening + GT. The samples of G2, G5, and G6 were whitened with $10 \%$ carbamide peroxide for $8 \mathrm{~h}$ daily, during 14 days at $37^{\circ} \mathrm{C}$. G3, G4, G5, and $\mathrm{G} 6$ were treated with the respective antioxidants (GT or SA) for $1 \mathrm{~h}$ at $37^{\circ} \mathrm{C}$. After the treatments, the brackets were bonded with Transbond XT. Then, the samples were tested in a universal testing machine and the adhesive remnant index (ARI) was examined under $40 x$ magnification. Data were submitted to analysis of variance, Tukey's and Dunnett's tests $(a=5 \%)$. The averages (standard deviations) obtained were: $G 1,17.6$ (4.1); $G 2,9.8$ (3.6); G3, 18.1 (4.1); G4, 18.2 (4.7); G5, 14.9 (2.5); and G6, 14.8 (5.4). G2 had statistically lower values than the other groups. Concerning the $\mathrm{ARI}$, all groups had a higher number of flaws type score 0 (no material adhered to the tooth). We conclude that the use of GT can be an alternative method to allow bracket bonding immediately after home whitening treatment.
\end{abstract}

Keywords: Tooth whitening, Carbamide peroxide, Brackets, Antioxidants

\section{Background}

In the fixed orthodontic therapy, it is essential to maintain the biomechanical stability of the bracket-adhesive interface, which transfers the force generated by the arches to the tooth [1]. Thus, when patients undergo whitening treatment before orthodontic treatment, a major concern is that the residues derived from peroxide or oxygen degradation leads to the formation of lower amounts of and smaller resin "tags" than in non whitened teeth $[2,3]$. This results in lower bonding strength, as this residual oxygen negatively affects the polymerization of the adhesive systems [4].

Nascimento et al. [5] recommend a waiting time of 3 weeks before bonding brackets on teeth that underwent whitening treatment. This time interval is often clinically impracticable, as some patients require immediate treatment and some do not want to wait. Thus, antioxidant substances to remove the residual oxygen from the tooth 
structure have been studied, including sodium ascorbate [6,7], catalase [8], alphatocopherol [9], grape seed extract [10], and, more recently, green tea [11, 12]. Although sodium ascorbate is the most studied antioxidant $[6,7,13]$, its use is still not clinically feasible. For this reason, further studies with alternative antioxidant substances such as green tea are required.

Polyphenolic compounds from green tea are antioxidants used in modern dentistry $[11,14]$. Green tea, which is extracted from the plant Camellia sinensis, contains various catechin compounds. Catechins are chemical antioxidants that have the ability to destroy free radicals. Epigallocatechin gallate (EGCG) is the most active and abundant catechin present in green tea, which is a water-soluble and a safe material [15]. Moreover, several studies have shown that the catechins in green tea are rapidly metabolized, which explains its antioxidant property [16].

Green tea, in a recent study [17], proved to be an alternative substance to restore the shear bonding strength in bleached enamel using a in-office technique. However, once the vital tooth bleaching procedures are the most commonly used conservative and effective treatments for discolored teeth [18], the use of substances which enable the bracket bonding immediately after the home whitening, are necessary.

Thus, this study compared the effect of $10 \%$ green tea and $10 \%$ sodium ascorbate on the shear bond strength of metal brackets after home treatment with $10 \%$ carbamide peroxide. The hypothesis tested was that green tea is able to reverse the shear bond strength of bracket to enamel after home bleaching treatment.

\section{Methods}

The study was submitted to and approved by the Ethics Committee under protocol \#354.444 (University of North Parana). In this study, 90 human premolars extracted for therapeutic reasons were selected. These premolars were healthy and with no restorations or composite resin from bracket bonding and enamel fractures. The teeth were stored for 7 days in a container with $0.5 \%$ chloramine-T solution for disinfection. Then, the teeth were washed in running water and cleaned with Mccall periodontal curettes, and prophylaxis was performed with extrafine pumice, with the aid of rubber cups.

The 90 premolars had their roots embedded in a ring inside a polyethylene mold for the crown buccal face to be in a perpendicular position to the horizontal level using autopolymerizing acrylic resin, therefore, only the crowns of the teeth remained exposed. Each buccal crown surface was pumiced with a rubber prophylaxis cup in a lowspeed conventional handpiece washed for $10 \mathrm{~s}$, and dried with a gentle continuous stream of oil-free compressed air. The samples were then divided into six experimental groups ( $\mathrm{n}=15$ each), as described below.

\section{Treatments}

Individual impression trays were made from silicon with a thickness of $1.0 \mathrm{~mm}$, manufactured with a vacuum laminator (Plastivac P7; BIOART, São Carlos, SP, Brazil), to carry out the whitening treatment and application of antioxidants.

The samples from groups 1,3 , and 4 were not subjected to whitening treatment. However, in groups 3 and 4, 10 \% sodium ascorbate gel (manufactured by Botica Chateau D'or, Maringá, PR, Brazil) and 10 \% green tea gel (manufactured from an extract 
containing $60 \%$ catechins and $5 \%$ caffeine by Botica Chateau D’or, Maringá, PR, Brazil), respectively, were applied to the vestibular surface of the samples (approximately $0.02 \mathrm{~mL}$ ) and an individual impression tray was positioned. The antioxidant was applied for $1 \mathrm{~h}$ at $37 \pm 1{ }^{\circ} \mathrm{C}$.

In groups 2,5 , and 6 , whitening treatment was performed, simulating a home whitening technique, by using $10 \%$ carbamide peroxide (Opalescence; Ultradent Inc., South Jordan, UT, USA). The gel was applied on the vestibular surface of the tooth and left for $6 \mathrm{~h}$ a day, for 14 days. During the $8 \mathrm{~h}$ of whitening and the $16 \mathrm{~h}$ of waiting, the samples were immersed in artificial saliva to the amelo-dentinal border at $37 \pm 1{ }^{\circ} \mathrm{C}$. Then the respective antioxidants were applied in groups 5 and 6 , as described above.

Immediately after the respective treatment in all groups, as described in Table 1, bonding of the brackets was performed. Before bonding, prophylaxis was performed again as described above, and then the brackets were bonded. Brackets for premolars were used (Orthometric, metal brackets Roth 0.022 in., Marília, SP, Brazil) and Transbond XT resin (3 M Unitek, South Peck, CA, USA), following the manufacturer's instructions. In each bracket, by using a tensiometer (Ormico, Glendora, CA, USA), a 450-g force was applied. Excess resin was removed with a dental explorer, and photopolymerization was performed (Radii Cal LED; SDI, Bayswater, Australia) for $40 \mathrm{~s}$ (10 s on each face: mesial, distal, occlusal, and cervical) with an intensity of $1200 \mathrm{~mW} / \mathrm{cm}^{2}$. After bonding, the samples were stored for $24 \mathrm{~h}$ in distilled/deionized water at $37^{\circ} \pm 1^{\circ} \mathrm{C}$.

\section{Shear bond strength test}

Twenty-four hours after bonding, the samples were tested in a universal testing machine (DL 2000; EMIC, São José dos Pinhais, Brazil). The chisel was positioned parallel to the vestibular surface of the tooth and bracket interface to allow force transmission in the occlusal-gingival direction. The machine was set at a speed of $0.5 \mathrm{~mm} / \mathrm{min}$ with a $50 \mathrm{kgf}$ load until the removal of the brackets. The results obtained were converted to MPa by dividing the values obtained (in Newton) by the bracket area $\left(11.35 \mathrm{~mm}^{2}\right)$.

\section{Analysis of the adhesive remnant index (ARI)}

Immediately after debonding, the enamel surface of each sample was examined in a stereoscopic magnifier (Bel Microimage Analyser; Bel Photonics, Monza, Italy) with 10x magnification, to determine the amount of adhesive remaining. The ARI was calculated according to the classification of Artun and Bergland [19], and modified by Meehan [20], with the following score definitions: 0 , no material adhered to the tooth; 1 , less than

Table 1 Division of the experimental groups

\begin{tabular}{lll}
\hline Group & Whitening & Antioxidant \\
\hline Group 1 & No & No \\
Group 2 & $10 \%$ Carbamide peroxide & No \\
Group 3 & No & $10 \%$ Sodium ascorbate \\
Group 4 & No & $10 \%$ Green tea \\
Group 5 & $10 \%$ Carbamide peroxide & $10 \%$ Sodium ascorbate \\
Group 6 & $10 \%$ Carbamide peroxide & $10 \%$ Green tea \\
\hline
\end{tabular}


half of the material adhered to the tooth; 2, more than half of the material adhered to the tooth; 3 , all the material adhered to the tooth, including the mesh impression of the bracket; EF, enamel fracture after debonding.

\section{Data analysis}

Data were tabulated, and the offset values were analyzed for normality by using the Shapiro-Wilk test, and then submitted to analysis of variance (ANOVA; two factors), considering the whitening and antioxidant factors. Tukey's test was then applied to verify the differences between the groups, and Dunnett's test was used to check the difference between the treated groups and the control groups. ARI values were compared by using the Kruskal-Wallis test followed by Dunn's test ( $\alpha=5 \%$ ).

\section{Results}

Table 2 shows the averages and standard deviations of the shear bond strength values. The analysis of power test was performed and indicated that at the $5 \%$ significance level, the test has a power of $99.5 \%$. ANOVA revealed no difference between the antioxidants tested $(\mathrm{p}=0.952)$, and interaction among the factors: whitening $\times$ antioxidant $(\mathrm{p}=0.916)$. However, there was a statistically significant difference for the whitening factor $(\mathrm{p}=0.004)$. Dunnett's test showed a statistically significant difference among the positive and negative control groups and the other groups $(\mathrm{p}<0.001)$.

Table 3 shows the analysis of the ARI. The statistical analysis showed no difference between the experimental groups $(\mathrm{p}=0.974)$; however, there was a statistically significant difference between the scores $(\mathrm{p}=0.001)$. Score 0 was statistically different from scores 2, 3, and EF.

Table 2 Average (standard deviation) according to the experimental groups $(\mathbf{n}=15)$

\begin{tabular}{lcc}
\hline Treatments & Sodium ascorbate & Green tea \\
\hline Without whitening & $18.07 \pm 4.10 \mathrm{Aa} *$ & $18.26 \pm 4.68 \mathrm{Aa}^{*}$ \\
With whitening & $14.85 \pm 2.49 \mathrm{Ab} *$ & $14.80 \pm 5.43 \mathrm{Ab}$ \\
Without whitening & $17.61 \pm 4.12^{*}$ & \\
Only whitened & $9.81 \pm 3.61^{\S}$ &
\end{tabular}

Averages followed by different capital letters differed statistically in Tukey's test $(p<0.05)$ comparing the antioxidants Averages followed by different lowercase letters differed statistically in Tukey's test $(p<0.05)$ for each antioxidant

$\S$ Statistically different from the positive control group in Dunnett's test $(p<0.05)$

* Statistically different from the negative control group in Dunnett's test $(p<0.05)$

Table 3 Adhesive remnant index score according to the experimental groups

\begin{tabular}{|c|c|c|c|c|c|c|c|c|c|}
\hline \multirow[t]{2}{*}{ Score } & \multicolumn{6}{|c|}{ Experimental group, $\mathrm{n}(\%)$} & \multirow[t]{2}{*}{$\mathbf{n}$} & \multirow[t]{2}{*}{ Dunn's test } & \multirow[t]{2}{*}{$\mathrm{p}$ value } \\
\hline & 1 & 2 & 3 & 4 & 5 & 6 & & & \\
\hline 0 & $15(100)$ & $14(93)$ & $11(73)$ & $6(40)$ & $14(93)$ & $15(100)$ & 15 & a & 0.001 \\
\hline 1 & $0(0)$ & $1(7)$ & $4(27)$ & $4(27)$ & $1(7)$ & $0(0)$ & 15 & $a b$ & \\
\hline 2 & $0(0)$ & $0(0)$ & $0(0)$ & $0(0)$ & $0(0)$ & $0(0)$ & 15 & $b$ & \\
\hline 3 & $0(0)$ & $0(0)$ & $0(0)$ & $0(0)$ & $0(0)$ & $0(0)$ & 15 & $b$ & \\
\hline EF & $0(0)$ & $0(0)$ & $0(0)$ & $5(33)$ & $0(0)$ & $0(0)$ & 15 & $\mathrm{~b}$ & \\
\hline
\end{tabular}




\section{Discussion}

In the present study, we evaluated the effect of the application of green tea and sodium ascorbate for $1 \mathrm{~h}$ after whitening with $10 \%$ carbamide peroxide, simulating a home whitening technique. The results showed that both antioxidants were able to reverse the shear bond strength values after whitening. Therefore, the hypothesis tested in this study was accepted.

The percentage of sites assigned an ARI scores of score 0 for all groups, indicating a poor interaction of the tooth structure with the bonding systems [21], which can result in low shear strength values. On the other hand, these results are clinically significant as it leaves the enamel surface relatively intact. According to Fox et al. [22], ideally in orthodontics, an adequate bond should fail at enamel composite interface (ARI $=0)$, as this would make as this would make deboning and subsequent polishing easier.

Scougall-Vilchis et al. [23] compared the influence of four tooth whitening systems on the shear bond strength of orthodontic brackets. The authors found that the use of a whitener containing $38 \%$ hydrogen peroxide did not significantly affect the shear bond strength values. However, the group treated with $10 \%$ carbamide peroxide showed a more significant decrease in strength than the control group (not whitened). These results corroborate those of the present study, as a significant decrease in the values of the negative control group (group 2) with the positive control group (group 1) was observed. These data confirm that home whitening treatment before the completion of bracket bonding negatively influences the bond strength of brackets $[3,7,24]$.

The results presented in Table 2 show that the application of the antioxidants tested was able to reverse the bond strength of the metal bracket. These findings corroborate the study of Berger et al. [11], which evaluated the application of green tea and sodium ascorbate, applied for $1 \mathrm{~h}$, on teeth whitened with $10 \%$ carbamide peroxide. The authors found that there was reversal of bond strength values of the enamel to the restorative composite. Moreover, the findings of Bulut et al. [7] also showed similar results to this study, with the use of $10 \%$ sodium ascorbate.

In contrast to this study, Uysal et al. [21] and Bishara et al. [25] reported that both the whitening treatment with $35 \%$ hydrogen peroxide and $10 \%$ carbamide peroxide did not affect the bond strength of the orthodontic bracket to the enamel. However, both studies suggest that it is prudent to wait around $2-3$ weeks before performing bracket bonding after the completion of whitening. Bishara et al. [25] reported that although there was no statistical difference between the groups treated with home and in-office whitening and the no whitened group, there was a large variation between the data. The authors tried to explain this phenomenon by using scanning electron microscopy images, as the enamel surface appeared uneven in the whitened groups.

Khamaverdi et al. [26] tested the application for 10 min of EGCG, the most active and abundant catechin present in green tea, and found that this time was sufficient to increase the shear bond strength values of the enamel to the composite resin. In the present study, we used the green tea with $60 \%$ catechins without standardization of the EGCG content. Thus, we speculate that EGCG is the main component for capturing free radicals, and, had we performed a standardization, a lower application time might have been required to obtain satisfactory results. However, future studies evaluating different types and concentrations of catechins to restore the shear bond strength between 
bonded orthodontics brackets and bleached enamel are needed to comprove this speculation.

The diverging results between studies testing antioxidants versus the reversal of bond strength values can be attributed to the different methods used in the studies. Kimyai et al. [13] applied sodium ascorbate in the form of hydrogel or solution for $10 \mathrm{~min}$ or $3 \mathrm{~h}$, and found that the application for $3 \mathrm{~h}$ obtained similar results to those of the present study, which used an application time of $1 \mathrm{~h}$. In the study by Bulut et al. [6, 7], sodium ascorbate solution was stirred on enamel surface during the 10-min application time, which may have increased its effectiveness. In the present study, a small amount of green tea or sodium ascorbate was placed on the enamel surface by using individual impression trays, simulating a possible clinical application.

Reynolds et al. [27] suggested that the appropriate minimum bond strength for most clinical needs, able to withstand masticatory forces and orthodontic forces, is 6-8 MPa. In the analysis of the values obtained in the present study, all groups showed average values $>9 \mathrm{MPa}$, proving that even samples subjected to whitening could withstand the stresses from orthodontic movement.

\section{Conclusion}

On the basis of the results obtained in this study, we can conclude that green tea is an alternative treatment capable of reversing the shear bond strength of the enamel bonding of brackets submitted to home whitening treatment with $10 \%$ carbamide peroxide.

Authors' contributions

RCAS, JSYL, AGJ and HCC participated in performing the experiments. MBL and RDG contributed to the writing of the manuscript. AS performed the statistical analysis. SBB conceived of the study, participating in its design and coordination, helping to drafting the manuscript. All authors read and approved the final manuscript.

\section{Author details}

${ }^{1}$ Department of Restorative Dentistry, University of North Parana, Rua Marselha, 183 Jardim Piza, Londrina, PR 86041-120, Brazil. ${ }^{2}$ Department of Orthodontics, Catholic University, City of the East, Paraguay.

\section{Competing interests}

The authors declare that they have no competing interests.

Received: 31 March 2016 Accepted: 28 June 2016

Published online: 04 July 2016

\section{References}

1. Eliades T, Brantley WA. The inappropriateness of conventional orthodontic bond strength assessment protocols. Eur J Orthod. 2000;22(1):13-23.

2. Gungor AY, Ozcan E, Alkis H, Turkkahraman H. Effects of different bleaching methods on shear bond strengths of orthodontic brackets. Angle Orthod. 2013;83(4):686-90.

3. Miles PG, Pontier JP, Bahiraei D, Close J. The effect of carbamide peroxide bleach on the tensile bond strength of ceramic brackets: an in vitro study. Am J Orthod Dentofacial Orthop. 1994;106(4):371-5.

4. Loretto SC, Braz R, Lyra AM, Lopes LM. Influence of photopolymerization light source on enamel shear bond strength after bleaching. Braz Dent J. 2004;15(2):133-7.

5. Nascimento GC, de Miranda CA, Machado SM, Brandao GA, de Almeida HA, Silva CM. Does the time interval after bleaching influence the adhesion of orthodontic brackets? Korean J Orthod. 2013;43(5):242-7.

6. Bulut $H$, Kaya AD, Turkun M. Tensile bond strength of brackets after antioxidant treatment on bleached teeth. Eur J Orthod. 2005:27(5):466-71.

7. Bulut $H$, Turkun M, Kaya AD. Effect of an antioxidizing agent on the shear bond strength of brackets bonded to bleached human enamel. Am J Orthod Dentofacial Orthop. 2006;129(2):266-72.

8. Torres CRGKA. Borges BA The effect of antioxidant agents as neutralizers of bleaching agents on enamel bond strength. Braz J Oral Sci. 2006;5(16):971-6. 
9. Sasaki RT, FI Rio FM, Basting RT. Effect of 10\% sodium ascorbate and 10\% ?-tocopherol in different formulations on the shear bond strength of enamel and dentin submitted to a home-use bleaching treatment. Oper Dent. 2009;34(6):746-52.

10. Vidhya S, Srinivasulu S, Sujatha M, Mahalaxmi S. Effect of grape seed extract on the bond strength of bleached enamel. Oper Dent. 2011;36(4):433-8.

11. Berger SB, De Souza Carreira RP, Guiraldo RD, Lopes MB, Pavan S, Giannini M, et al. Can green tea be used to reverse compromised bond strength after bleaching? Eur J Oral Sci. 2013;121(4):377-81.

12. Ozelin AA, Guiraldo RD, Carvalho RV, Lopes MB, Berger SB. Effects of green tea application time on bond strength after enamel bleaching. Braz Dent J. 2014;25(5):399-403.

13. Kimyai S, Oskoee SS, Rafighi A, Valizadeh H, Ajami AA, Helali ZN. Comparison of the effect of hydrogel and solution forms of sodium ascorbate on orthodontic bracket-enamel shear bond strength immediately after bleaching: an in vitro study. Indian J Dent Res. 2010;21(1):54-8.

14. Magalhaes AC, Wiegand A, Rios D, Hannas A, Attin T, Buzalaf MA. Chlorhexidine and green tea extract reduce dentin erosion and abrasion in situ. J Dent. 2009;37(12):994-8.

15. Lambert JD, Elias RJ. The antioxidant and pro-oxidant activities of green tea polyphenols: a role in cancer prevention. Arch Biochem Biophys. 2010;501(1):65-72.

16. Frei B, Higdon JV. Antioxidant activity of tea polyphenols in vivo: evidence from animal studies. J Nutr. 2003;133(10):3275S-84S.

17. Berger SB, Guiraldo RD, Lopes MB, Oltramari-Navarro PV, Fernandes TM, Schwertner Rde C, et al. Effects of green tea on the shear bond strength of orthodontic brackets after in-office vital bleaching. Gen Dent. 2016;64(3):72-5.

18. Cavalli V, de Carvalho RM, Giannini M. Influence of carbamide peroxide-based bleaching agents on the bond strength of resin-enamel/dentin interfaces. Braz Oral Res. 2005;19(1):23-9.

19. Artun J, Bergland S. Clinical trials with crystal growth conditioning as an alternative to acid-etch enamel pretreatment. Am J Orthod. 1984;85(4):333-40.

20. Meehan MP, Foley TF, Mamandras AH. A comparison of the shear bond strengths of two glass ionomer cements. Am J Orthod Dentofacial Orthop. 1999;115(2):125-32.

21. Uysal T, Basciftci FA, Usumez S, Sari Z, Buyukerkmen A. Can previously bleached teeth be bonded safely? Am J Orthod Dentofacial Orthop. 2003;123(6):628-32.

22. Fox NA, McCabe JF, Buckley JG. A critique of bond strength testing in orthodontics. Braz J Orthod. 1994;21(1):33-43.

23. Scougall-Vilchis RJ, Gonzalez-Lopez BS, Contreras-Bulnes R, Rodriguez-Vilchis LE, Garcia-Nino de Rivera MW, Kubodera-Ito T. Influence of four systems for dental bleaching on the bond strength of orthodontic brackets. Angle Orthod. 2011;81(4):700-6.

24. Machado SM, Nascimento DB, Silva RC, Loretto SC, Normando D. Evaluation of metallic brackets adhesion after the use of bleaching gels with and without amorphous calcium phosphate (ACP): in vitro study. Dental Press J Orthod. 2013;18(3):101-6.

25. Bishara SE, Oonsombat C, Soliman MM, Ajlouni R, Laffoon JF. The effect of tooth bleaching on the shear bond strength of orthodontic brackets. Am J Orthod Dentofacial Orthop. 2005;128(6):755-60.

26. Khamverdi Z, Rezaei-Soufi L, Kasraei S, Ronasi N, Rostami S. Effect of Epigallocatechin Gallate on shear bond strength of composite resin to bleached enamel: an in vitro study. Restor Dent Endod. 2013;38(4):241-7.

27. Reynolds IR. A review of direct orthodontic bonding. Braz J Orthod. 1975;2:8.

\section{Submit your manuscript to a SpringerOpen ${ }^{\circ}$ journal and benefit from:}

- Convenient online submission

- Rigorous peer review

- Immediate publication on acceptance

- Open access: articles freely available online

- High visibility within the field

- Retaining the copyright to your article

Submit your next manuscript at $>$ springeropen.com 